\title{
Trabajo por niveles de desempeño cognitivo en el contexto ecuatoriano: ¿Alternativa o necesidad?
}

\author{
Work by levels of cognitive performance in the Ecuadorian context: Alternative or \\ need?
}

\author{
Yullio Cano de la Cruz \\ e-mail: ccy@pucesd.edu.ec \\ Pontificia Universidad Católica del Ecuador. Ecuador
}

\author{
Edgar Efraín Obaco Soto \\ e-mail: osef@pucesd.edu.ec \\ Pontificia Universidad Católica del Ecuador. Ecuador
}

\author{
Lianet Delgado Mendoza \\ e-mail: fisioterapia.lia@gmail.com \\ Pontificia Universidad Católica del Ecuador. Ecuador
}

\author{
Génesis Nicole Herrera Orosco iD \\ e-mail: gnherrerao@pucesd.edu.ec \\ Pontificia Universidad Católica del Ecuador. Ecuador
}

José Marcelino Romero Gutierrez

e-mail: rgimp@ucesd.edu.ec

Pontificia Universidad Católica del Ecuador. Ecuador

\section{Resumen}

El desarrollo cognitivo es el conjunto de procesos a través de los cuales el ser humano adquiere habilidades que le permiten interpretar la realidad e interactuar con ella de una forma eficiente, es decir, es la evolución de las capacidades intelectuales que le permite resolver problemas. Desde esta perspectiva, el presente estudio tuvo como objetivos: identificar el nivel de desempeño cognitivo de un grupo de estudiantes de sexto grado; determinar el nivel de conocimientos que tienen los docentes acerca de los estudios de calidad y el trabajo por niveles de desempeño cognitivo; y ofrecer recomendaciones metodológicas que sirvan de orientación para el desarrollo de estos niveles. Se utilizó una metodología cuantitativa, siendo un estudio de alcance exploratorio. Se seleccionaron dos muestras. La primera integrada por 55 docentes, a los que se les aplicó una encuesta; y la segunda compuesta de 117 estudiantes de sexto grado a los que se les aplicaron tres cuestionarios. Los resultados demostraron que el mayor porcentaje de los estudiantes se ubica en el nivel más bajo de desempeño cognitivo (primer nivel), mientras que los docentes tienen un deficiente conocimiento acerca de los estudios de calidad educativa y el trabajo por niveles de desempeño cognitivo se realiza con poca frecuencia y de manera aislada, lo que generó la necesidad de ofrecer un conjunto de recomendaciones metodológicas sustentadas en el paradigma cognitivo o de procesamiento de la información.

Palabras clave: cognición; aprendizaje; educación; desarrollo de las habilidades; calidad de la educación.

\section{Abstract}

Cognitive development is the set of processes through which human beings acquire skills that allow them to interpret reality and interact with it in an efficient way, that is, the evolution of intellectual capacities that allows them to solve problems. From this perspective, the present study had as objectives: to identify the level of cognitive performance of a group of sixth grade students; determine the level of knowledge that teachers have about quality studies and work by levels of cognitive performance; and offer methodological recommendations that serve as guidance for the development of these levels. This being an exploratory study, a quantitative methodology was used. Two samples were selected. The first made up of 55 teachers, to whom a survey was applied; and the second, made up of 117 sixth grade students to whom three questionnaires were applied. The results showed that the largest percentage of students is located in the lowest level of cognitive performance (first level), while teachers have poor knowledge about educational quality studies, and work by levels of cognitive performance is carried out infrequently and in isolation, which generated the need to offer a set of methodological recommendations based on the cognitive or information-processing paradigm.

Keywords: cognition; learning; level of education; instruction; skills development; quality of education.

Recibido / Received: 04-04-2020

Aceptado / Accepted: 02-04-2021

Publicación en linea / Published online: 28-07-2021

Cómo referenciar este artículo / How to reference this article:

Cano de la Cruz, Y., Obaco Soto, E. E., Delgado Mendoza, L., Herrera Orosco, G. N., \& Romero Gutierrez, J. M. (2021). Trabajo por niveles de desempeño cognitivo en el contexto ecuatoriano: ¿Alternativa o necesidad? Tendencias Pedagógicas, 38, pp. 112-123. doi: 10.15366.tp2021.38.010. 


\section{Introducción}

El desarrollo cognitivo es un proceso de incremento de los conocimientos y habilidades que va aparejado a la maduración de las funciones cognitivas y ejecutivas: atención, memoria, percepción, control inhibitorio y flexibilidad cognitiva. Es de gran importancia para el individuo, pues le permite resolver problemáticas que se le presenten en la vida. Este desarrollo comienza desde los primeros años de vida, siendo importante que se potencie a través de la estimulación temprana (Albornoz, Zamora y Guzmán, 2016). Sin embargo, a criterio de Papalia, et al., (2012), este proceso continúa en las siguientes etapas del desarrollo del individuo, por lo que estos autores resaltan la importancia de su constante estimulación.

En este sentido, aun cuando la bibliografía consultada se inclina por la familia como el principal factor de incidencia en el desarrollo cognitivo, Alves, et al., (2017); Terranova, et al., (2019); Treviño et al., (2016), también se destaca el papel de la escuela, siendo diversas las investigaciones en el ámbito de la educación que señalan la importancia de la calidad de la docencia y la efectividad de los procesos de enseñanza-aprendizaje como factores claves para mejorar el desempeño cognitivo de los individuos (Carnoy, 2007); (Pianta et al., 2011); Vaillant, (2006). La interacción en clase bajo un adecuado ambiente de aprendizaje promueve la estimulación cognitiva y lingüística (Hunt, 2009); (Pianta et al., 2011).

Estos aspectos destacan la importancia de las instituciones educativas en el desarrollo cognitivo de los individuos, que necesita ser estimulado desde los primeros años de vida a través de una serie de actividades y experiencias de aprendizaje que les permitan transitar por cada uno de los niveles de desempeño con miras a un desarrollo cognitivo superior.

Por su importancia y la necesidad de su desarrollo desde la escuela, el desempeño cognitivo se convierte en un indicador de calidad de la educación utilizado por organismos internacionales asociados a la Organización de las Naciones Unidas para la Educación, la Ciencia y la Cultura (UNESCO, por sus siglas en inglés) encargados del monitoreo de la calidad educativa.

En América Latina, el Laboratorio Latinoamericano de Evaluación de la Calidad de la Educación (LLECE), a través de la Oficina Regional de Educación para América Latina y el Caribe, con sede en Chile, trabaja en el seguimiento del Objetivo de Desarrollo Sostenible N. 4 del Marco de Acción de la Agenda de Educación 2030, sobre educación en la región. Para ello, este Laboratorio ha realizado cuatro estudios regionales comparativos y explicativos de la calidad de la educación, basados en la medición de los niveles de desempeño cognitivo.

El último estudio realizado fue en el año 2019, denominado ERCE (Cuarto Estudio Regional y Comparativo de la Calidad de la Educación), sin embargo, sus resultados saldrán a finales del 2020, principios del 2021. Anteriormente, los resultados del Tercer Estudio Regional y Comparativo de la Calidad de la Educación, TERCE, (2013), en el que participaron 15 países de la región a los que se sumó el Estado de Nuevo León, México, mostraron un panorama nada alentador en cuanto al desempeño cognitivo de los estudiantes de la muestra.

Los resultados de este estudio se muestran desde dos perspectivas: la primera mediante puntajes representados por una métrica cuya media es de 500 puntos y que tiene una desviación estándar de 100; y la segunda mediante cuatro niveles de desempeño cognitivo, donde los niveles I y II son deficientes y el III y IV los más altos. Incluso existe un nivel inferior para aquellos estudiantes que no se pueden ubicar en ningún nivel. Esos niveles describen lo que saben y saben hacer los estudiantes en función del currículo, las metas y resultados de aprendizaje; están referidos al grado de procesamiento intelectual o a la demanda cognoscitiva involucrada en la resolución de problemas (Ineval, 2014).

En el caso de las puntuaciones promedio, estas fueron de 698 en el tercer grado y de 683 en el sexto grado, de un límite máximo de 1000 puntos. Estas cifras ubican a Ecuador cerca de la media regional, lo que muestra una paridad entre los países que participaron en el estudio (Flotts, et al., 2016).

En cuanto a las puntuaciones por asignatura, en el caso de Matemática, se tomó una muestra de 4642 estudiantes de tercer grado y la misma cantidad de estudiantes de sexto grado, los cuales obtuvieron una puntuación promedio de 703 y 702 respectivamente, ubicando al Ecuador en la media regional (Flotts et al., 2016).

Similar puntuación se obtuvo en la asignatura de Ciencias Naturales, con una media de 711 puntos que corresponde a los 4820 estudiantes evaluados. En el caso de la escritura la situación es distinta, con un promedio de 280 puntos para los 4621 estudiantes de tercer grado y 249 puntos alcanzados 
por los 4826 estudiantes de sexto grado; lo que indica un nivel de desempeño cognitivo inferior en comparación con el resto de países participantes, que ubica al Ecuador por debajo de la media regional (Flotts et al., 2016).

En cuanto a la clasificación por niveles de desempeño cognitivo, el Ecuador concentró el mayor porcentaje de estudiantes en el primer y segundo nivel, lo que mostró deficiencias en la interpretación de información más compleja, aplicación de conocimientos científicos, análisis, y resolución de problemas, entre otras que se consideran esenciales (Flotts, et al., 2016).

A esto se le añade que los estudiantes de 15 años que participaron en las pruebas PISA-D (Programa para la Evaluación Internacional de Alumnos, 2017) obtuvieron resultados similares, considerando que, si bien Ecuador fue el país más destacado, la mayor parte de los estudiantes o llegaron al segundo nivel en Matemática y Ciencias, BBC Mundo, Agencias-Redacción (2019), lo que indica que el bajo desempeño cognitivo de los estudiantes ecuatorianos se presenta en todos los grupos etarios.

Ante este panorama, si bien es cierto que el Ministerio de Educación ecuatoriano (MINEDUC) ha tomado medidas encaminadas a revertir dicha situación, como son las Reformas Curriculares realizadas en el 2010 y 2016 y el establecimiento de Estándares de Calidad Educativa, todavía persisten deficiencias que impiden que los estudiantes logren el tránsito por los diferentes niveles de desempeño cognitivo.

De acuerdo con el análisis de las causas del bajo desempeño cognitivo presentado por el TERCE, se demostró que las variables con mayor incidencia fueron las vinculadas con la percepción de los estudiantes sobre el clima de aula y la actuación de los docentes, lo que reveló la importancia que debe darse a la formación y capacitación de los docentes (Treviño, E., et al., 2016).

Otras investigaciones sugieren que un nivel educativo alto generalmente se asocia a un mejor desempeño cognitivo (Thow et al., 2018) y con el desarrollo de habilidades (Harrison et al., 2015, como se citó en Feldberg, et al., 2020), por lo que resulta interesante conocer cómo trabajan en el desarrollo de los niveles de desempeño cognitivo los docentes ecuatorianos.

Desde esta perspectiva, los propósitos del presente estudio son los siguientes:

Objetivo 1: identificar el nivel de desempeño cognitivo de los estudiantes de las instituciones educativas de Santo Domingo de los Tsáchilas.

Objetivo 2: determinar el nivel de conocimiento que tienen los docentes de las instituciones educativas de Santo Domingo de los Tsáchilas acerca de los estudios de la calidad de la educación y el trabajo por niveles de desempeño cognitivo.

Objetivo 3: ofrecer un conjunto de recomendaciones, sustentadas en el paradigma cognitivo o de procesamiento de la información para el trabajo por niveles de desempeño cognitivo en las aulas ecuatorianas.

\subsection{Aproximación teórica a los niveles de desempeño cognitivo}

Realizar una aproximación teórica al término «niveles de desempeño cognitivo» implica diferenciar entre las similitudes que tiene con los niveles de asimilación del contenido y el desarrollo cognitivo. A criterio de Tundidor (2019), en la actualidad se observa una tendencia al desuso de la categoría niveles de asimilación del conocimiento, alegando que se ha sustituido por la de niveles de desempeño cognitivo. Si bien es cierto, es importante destacar que los niveles de asimilación en la actualidad están referidos más al plano didáctico, al nivel de profundidad con que deberá manejar el docente los contenidos en el aula y trabajar en función de estos (Ruvalcaba, 2015).

Por su parte, el desarrollo cognitivo, para Ordoñez y Tinajero (2005), como se citó en Albornoz Zamora y Guzmán (2016), es un proceso que comienza desde los primeros años, mediante el cual el niño organiza la información senso-perceptual que recibe para resolver situaciones nuevas en base a experiencias pasadas.

A medida que se incrementa la estimulación durante el proceso de crecimiento y maduración, el individuo logra diferentes estadios en su desarrollo cognitivo, los cuales pueden categorizarse de acuerdo con el grado de desarrollo alcanzado en lo que se conoce como niveles de desempeño cognitivo (Rubio, 2006). Para Puig (2003), estos niveles son la manifestación externa que realiza una persona en el cumplimiento de una tarea, ya sea intelectual y/o práctica. De manera sintética, se pueden definir como el grado de desarrollo cognitivo logrado en los estudiantes en el que se manifiestan los conocimientos, las habilidades y los valores. Estos se dividen, según Puig (2003), en primer nivel de desempeño cognitivo o reproductivo, segundo nivel o aplicativo y tercer nivel o creativo. 
Según Rojas y Camejo (2009), el primer nivel o reproductivo se caracteriza, como su denominación indica, por actividades de reproducción del objeto del conocimiento en base a la memoria, así exista un proceso de comprensión o no. En este nivel, según Puig (2003), el estudiante debe demostrar su capacidad para utilizar las operaciones de carácter instrumental que son básicas en una asignatura dada, para ello deberá reconocer, identificar, describir e interpretar los conceptos y propiedades esenciales en los que esta se sustenta.

En el segundo nivel de desempeño cognitivo o de aplicación su capacidad deberá estar dirigida a establecer relaciones conceptuales, donde además de reconocer, describir e interpretar los conceptos deberá aplicarlos a una situación planteada y reflexionar sobre sus relaciones internas. Por último, en el tercer nivel de desempeño cognitivo o nivel de creación el estudiante demostrará resolver problemas en los que reconozca la situación problemática, identifique los componentes e interrelaciones, establezca estrategias de solución, además de fundamentar o justificar lo realizado (Puig, 2003).

Para la medición de los niveles de desempeño cognitivo se indica el procedimiento descrito por Leyva, et al., (2008). Siguiendo este criterio, para que un estudiante se ubique en un primer nivel de desempeño cognitivo o nivel reproductivo, debe responder el $60 \%$ de las preguntas, reactivo o tareas de aprendizaje correspondientes al primer nivel.

La obtención del primer nivel es prerrequisito para el segundo, a lo que se suma responder mínimo el $50 \%$ de las interrogantes de este nivel y, en el caso del tercer nivel, se requiere de los dos niveles anteriores más el $40 \%$ de las interrogantes planteadas.

Esta tendencia netamente cuantitativa es criticada en el ámbito académico, sin embargo, es una herramienta eficiente para determinar en qué nivel se encuentra el estudiante, además, es un punto referencial que el docente verifique el tránsito por los niveles de asimilación para lograr establecer el desarrollo cognitivo del estudiante.

\subsection{Sustento epistemológico de las recomendaciones metodológicas: el paradigma cognitivo o de procesamiento de la información}

Como sustento epistemológico de las recomendaciones metodológicas que se ofrecen, se tomó el paradigma cognitivo o de procesamiento de la información (De Vega, 1984); (Gardner, 1987); (Pozo, 1989). Este paradigma orienta la educación a lograr el desarrollo de habilidades, partiendo de la idea de que el aprendizaje de contenidos o dominios del conocimiento no es suficiente.

El sujeto debe desarrollar una serie de habilidades intelectuales que le permitan conducirse ante cualquier tipo de situaciones de aprendizaje y aplicar los conocimientos adquiridos frente a situaciones complejas y que desarrolle su potencialidad cognitiva convirtiéndose en un aprendiz estratégico (Ausubel, 1975). Desde esta perspectiva, las metas de la escuela deben centrase en el aprender a aprender, de manera combinada con el enseñar a pensar, para que los sujetos egresen como procesadores activos y efectivos del conocimiento (Nickerson, Perkins y Smith, 1987).

Desde lo metodológico se parte del criterio de Cooper (1999), como se citó en Villalobos (2003), quien indica que se deben proponer actividades de aprendizaje que permitan al estudiante aprender en varios ámbitos, dentro de los cuales se encuentra el cognitivo, donde se dará importancia a la transferencia. El aprendizaje es visto como un sistema de instrucción dirigido por el docente Barragán (2007), por lo que se deben implementar estrategias instruccionales centradas en promover el aprendizaje significativo que activen o desarrollen los conocimientos previos.

En el proceso metodológico deben primar las estrategias de procesamiento de la información, donde se potencie la comprensión, la retención, la aplicación y la resolución de problemas, entre otras, desde la definición de conocimientos básicos específicos con base a mediadores y puentes de cognición que permitan la transferencia (Coll et al., 1995).

Este paradigma, al centrarse en los procesos de aprendizaje del aprendiente, debe considerar una evaluación centrada en las habilidades y estrategias básicas que el estudiante domina y los modelos conceptuales que posee (Blanco Gutiérrez, 2003).

La evaluación, más que un cuestionario de preguntas objetivas debe ser formativa que le permita al alumno detectar sus propios errores. Esta se debe diseñar en función de que mida los niveles de desempeño cognitivo desarrollados en los estudiantes. Para ello debe desarrollarse a través de pruebas escritas individuales en las que se combinen preguntas o ítems cerrados con otras de carácter abierto o de ensayo que permitan medir los contenidos factuales, conceptuales y procedimentales (Rossi, et 
al., 2009). Además, a criterio de estos autores se deben combinar otras técnicas de evaluación como las exposiciones orales, los estudios de caso y los informes de investigación.

\section{Desarrollo}

\subsection{Materiales y métodos}

La metodología utilizada en el estudio se sustentó en el paradigma cuantitativo, siendo de tipo exploratorio con un diseño transversal.

Participantes

Para el desarrollo del estudio se seleccionaron dos tipos de muestra. La primera consistió en una muestra aleatoria de 117 estudiantes de sexto grado de cuatro unidades educativas del cantón Santo Domingo. La segunda fue una muestra probabilística aleatoria consistente en 56 docentes de ocho Unidades Educativas del Cantón Santo Domingo, de la provincia homónima en Ecuador. El criterio de selección fue la voluntariedad de participar en el estudio. En lo que respecta a la distribución de la muestra en función de la variable titulación es importante destacar que $32(57,14 \%)$ tienen titulación de tercer nivel en el campo amplio de Educación y el resto en otros campos del conocimiento, pero todos cuentan con más de cinco años de experiencia en la docencia.

$V$ ariable y mediciones. Instrumentos empleados

La primera variable medida fue el desempeño cognitivo de los estudiantes. Para ello se aplicaron tres cuestionarios: uno del área de matemática, uno de lengua y literatura y uno de ciencias naturales.

La segunda variable fue el conocimiento acerca de los niveles de desempeño cognitivo y su trabajo en el aula. Esta variable se operacionalizó en dos dimensiones: conocimiento y trabajo. La dimensión conocimiento tuvo como indicadores: conocimiento acerca del LLECE y los operativos de calidad; conocimientos acerca de los niveles de desempeño cognitivo y vías por las que obtiene la información. La segunda dimensión, trabajo en el aula, tuvo como indicadores: frecuencia que trabaja por niveles de desempeño cognitivo y nivel de desempeño cognitivo que trabaja en el aula. A la muestra seleccionada se le administró, como instrumento de recogida de datos, una encuesta elaborada por los autores. Independientemente de que los instrumentos utilizados en el estudio fueron de carácter ad hoc, fueron sometidos a validación por criterio de jueces y expertos.

Para lo cual, en primer lugar, se procedió a verificar su validez de contenido mediante el envío a siete jueces y expertos, cuya valoración permitió calcular la Razón de Validez de Contenido (RVC, Lawshe, 1986). Una vez determinada la Razón, el índice obtenido se interpretó mediante el criterio de Davis (1992), a partir de lo cual quedaron en los instrumentos definitivos solo los ítems que tuvieron un índice superior a 0,80 .

En el caso de los instrumentos que les fueron administrados a los estudiantes, estos estuvieron compuestos por 12 interrogantes, cuatro por cada nivel de desempeño cognitivo; en tanto que el instrumento definitivo que se le aplicó a los docentes quedó compuesto por 10 ítems. De ellos, siete fueron dicotómicos, con un nivel de medición nominal y tres de tipo pregunta cerrada en abanico, con un nivel de medición ordinal. El análisis de los datos se realizó utilizando la estadística inferencial, específicamente la distribución de frecuencia en tablas y el cálculo porcentual. Como medios se utilizó el paquete de Microsoft Excel.

Procedimiento de recogida y análisis de datos

En primer lugar, se solicitaron los permisos correspondientes para administrar los cuestionarios a los estudiantes y se constató la voluntariedad de participar en el estudio de los docentes. Posteriormente, se les informó de los objetivos del mismo, el anonimato y la confidencialidad. El procedimiento consistió en la aplicación directa del cuestionario de la encuesta, la cual se les entregó de manera física a los participantes en una sola sesión y sin límites de tiempo.

\subsection{Resultados}

Nivel de desempeño cognitivo de los estudiantes de las instituciones educativas del cantón Santo Domingo El primer resultado está en función del objetivo: identificar el nivel de desempeño cognitivo de los estudiantes de las instituciones educativas de Santo Domingo de los Tsáchilas. Como se muestra en la tabla 1, el mayor número de estudiantes responde de manera incorrecta o deja en blanco las interrogantes planteadas. 
Tabla 1.

Total de respuestas correctas

\begin{tabular}{llllll}
\hline Correctas & $\mathbf{\%}$ & Incorrectas & $\mathbf{\%}$ & En blanco & $\mathbf{\%}$ \\
\hline $\mathbf{1 0 5 5}$ & 29,26 & 1767 & 49,03 & 782 & 21,7
\end{tabular}

Fuente: elaboración propia.

En la tabla 2 se aprecia un porcentaje de respuestas correctas solo en las correspondientes al primer nivel.

Tabla 2.

Respuestas correctas por niveles de desempeño

\begin{tabular}{lllllll}
\hline & Correctas & $\mathbf{0}$ & Incorrectas & $\mathbf{\%}$ & En blanco & $\mathbf{\%}$ \\
\hline I NIVEL & 576 & 54,60 & 389 & 22,01 & 145 & 18,54 \\
\hline II NIVEL & 322 & 30,52 & 883 & 49,97 & 266 & 30,02 \\
\hline III NI- & 157 & 14,88 & 495 & 28,01 & 371 & 47,44
\end{tabular}

VEL

Fuente: elaboración propia.

Por último, en la tabla 3 se puede precisar que el mayor número de estudiantes se encuentra en el primer nivel de desempeño cognitivo.

Tabla 3.

Estudiantes por niveles de desempeño cognitivo

\begin{tabular}{llllll}
\hline I Nivel & $\mathbf{\%}$ & II Nivel & $\mathbf{\%}$ & III Nivel & $\mathbf{\%}$ \\
\hline $\mathbf{6 7}$ & 63,21 & 21 & 19,81 & 18 & 16,98 \\
\hline
\end{tabular}

Fuente: elaboración propia.

Nivel de conocimientos acerca de los niveles de desempeño cognitivo y su empleo en el aula

Este resultado estuvo en correspondencia con el segundo objetivo, encaminado a determinar el nivel de percepción que tienen los docentes de las instituciones educativas de Santo Domingo de los Tsáchilas acerca de los estudios de la calidad de la educación y el trabajo por niveles de desempeño cognitivo, para ello se administró una encuesta a la misma muestra, que arrojó los siguientes datos (tablas 3 y 4):

Tabla 4.

Resultados obtenidos en la dimensión 1 conocimiento

\begin{tabular}{|c|c|c|c|c|c|}
\hline Ítem & Opciones & f & $\%$ & $\mathbf{f}^{\prime}$ & $\%$ \\
\hline \multirow{3}{*}{$\begin{array}{l}\text { ¿Conoce usted el LLECE y sus } \\
\text { funciones? }\end{array}$} & Sí & 14 & 25 & 0,25 & 25 \\
\hline & No & 42 & 75 & 0,75 & 75 \\
\hline & Total & 56 & 100 & 1 & 100 \\
\hline \multirow{3}{*}{$\begin{array}{l}\text { ¿Conoce usted algunos de los re- } \\
\text { sultados obtenidos por el Ecuador } \\
\text { en los Estudios Regionales, Expli- } \\
\text { cativos y Comparativos de la Cali- } \\
\text { dad de la Educación? }\end{array}$} & Sí & 27 & 48,21 & 0,48 & 48,21 \\
\hline & No & 29 & 51,79 & 0,52 & 51,79 \\
\hline & Total & 56 & 100 & 1 & 100 \\
\hline \multirow{5}{*}{$\begin{array}{l}\text { ¿Considera usted que el MI- } \\
\text { NEDUC establece acciones dirigi- } \\
\text { das a elevar los resultados obteni- } \\
\text { dos en los Estudios Regionales, } \\
\text { Explicativos y Comparativos de la } \\
\text { Calidad de la Educación? }\end{array}$} & Sí & 22 & 39,29 & 0,39 & 39,29 \\
\hline & No & 34 & 60,71 & 0,61 & 60,71 \\
\hline & Total & 56 & 100 & 1 & 100 \\
\hline & Sí & 22 & 39,29 & 0,39 & 39,29 \\
\hline & No & 34 & 60,71 & 0,61 & 60,71 \\
\hline
\end{tabular}




\begin{tabular}{|c|c|c|c|c|c|}
\hline $\begin{array}{l}\text { ¿Conoce usted los niveles de } \\
\text { desempeño cognitivo y cómo tra- } \\
\text { bajarlos en clase? }\end{array}$ & Total & 56 & 100 & 1 & 100 \\
\hline \multirow{5}{*}{$\begin{array}{l}\text { ¿Por dónde recibió usted informa- } \\
\text { ción acerca del LLECE y sus estu- } \\
\text { dios? }\end{array}$} & $\begin{array}{l}\text { A través de la } \\
\text { prensa }\end{array}$ & 11 & 57,14 & 0,57 & 57,14 \\
\hline & $\begin{array}{l}\text { A través de bole- } \\
\text { tines informati- } \\
\text { vos del MI- } \\
\text { NEDUC }\end{array}$ & 0 & 7,14 & 0,07 & 7,14 \\
\hline & $\begin{array}{l}\text { A través de capa- } \\
\text { citaciones del } \\
\text { MINEDUC }\end{array}$ & 0 & 12,50 & 0,13 & 12,50 \\
\hline & Otros & 33 & 23,21 & 0,23 & 23,21 \\
\hline & Total & 56 & 100 & 1 & 100 \\
\hline
\end{tabular}

Fuente: elaboración propia.

Al observar los datos mostrados en la tabla 3 es notable destacar que solo entre un 25 y un $40 \%$ conocen acerca del LLECE y sus estudios, además, por los porcentajes alcanzados, unido a la revisión de la oferta de cursos de capacitación impartidos por el ministerio, se puede inferir que el MINEDUC no ha divulgado los resultados, además, aun cuando el 39,29\% de la muestra considera que esta entidad realiza acciones en función de mejorar los resultados obtenidos, la práctica plantea que no es así, al no divulgar los resultados y no desarrollar capacitaciones al respecto.

En cuanto a la segunda dimensión, trabajo por niveles de desempeño cognitivo en el aula, se obtuvieron los siguientes resultados (tabla 5):

Tabla 5.

Resultados obtenidos en la dimensión 2 trabajo en el aula

\begin{tabular}{|c|c|c|c|c|c|}
\hline Ítem & Opciones & f & $\%$ & f & $\%$ \\
\hline \multirow{3}{*}{$\begin{array}{l}\text { ¿Trabaja usted en sus clases } \\
\text { por niveles de desempeño } \\
\text { cognitivo? }\end{array}$} & Sí & 17 & 30,36 & 0,30 & 30,36 \\
\hline & No & 39 & 69,64 & 0,70 & 69,64 \\
\hline & Total & 56 & 100 & 1 & 100 \\
\hline \multirow{4}{*}{$\begin{array}{l}\text { En caso de responder afirma- } \\
\text { tivamente: ¿Con qué frecuen- } \\
\text { cia usted trabaja los niveles de } \\
\text { desempeño cognitivo? }\end{array}$} & Siempre & 0 & 0,00 & 0,00 & 0,00 \\
\hline & Casi Siempre & 12 & 21,43 & 0,21 & 21,43 \\
\hline & A veces & 5 & 8,93 & 0,09 & 8,93 \\
\hline & Total & 17 & 30,36 & 0,30 & 30,36 \\
\hline \multirow{5}{*}{$\begin{array}{l}\text { En caso de responder afirma- } \\
\text { tivamente el ítem } 5: ¿ Q \text { ué ni- } \\
\text { vel de desempeño cognitivo } \\
\text { trabaja usted en clase? }\end{array}$} & Primer nivel & 5 & 8,93 & 0,09 & 8,93 \\
\hline & Segundo nivel & 2 & 3,57 & 0,04 & 3,57 \\
\hline & Tercer nivel & 3 & 5,36 & 0,05 & 5,36 \\
\hline & $\begin{array}{l}\text { Todos de manera inte- } \\
\text { grada }\end{array}$ & 7 & 12,50 & 0,13 & 12,50 \\
\hline & Total & 17 & 30,36 & 0,30 & 30,36 \\
\hline \multirow{3}{*}{$\begin{array}{l}\text { Por último: ¿Considera usted } \\
\text { necesario recomendaciones } \\
\text { metodológicas para el trabajo } \\
\text { por niveles de desempeño } \\
\text { cognitivo en clases? }\end{array}$} & Sí & 47 & 83,93 & 0,84 & 83,93 \\
\hline & No & 9 & 16,07 & 0,16 & 16,07 \\
\hline & Total & 56 & 100 & 1 & 100 \\
\hline
\end{tabular}

Fuente: elaboración propia.

En esta dimensión es notable destacar que solo un $30 \%$ aproximadamente trabaja por niveles de desempeño cognitivo en el aula, y solo un 12,5\% de este $30 \%$ los trabaja de manera integrada, el 
resto solo se centra en un nivel. Sin embargo, es significativo destacar que, de la totalidad de la muestra, el 83,93\% sí considera necesario que se hagan recomendaciones metodológicas, por lo que se puede inferir la predisposición para revertir la situación descrita.

Recomendaciones metodológicas para el trabajo por niveles de desempeño cognitivo

En correspondencia con los datos obtenidos se planteó como objetivo un conjunto de recomendaciones metodológicas, las cuales surgen de la experiencia de los autores y la revisión bibliográfica desarrollada; así, la primera recomendación está dirigida a que el docente diagnostique en qué nivel de desempeño cognitivo se encuentran sus estudiantes, de manera que le sirva de punto de partida para ir proyectando las actividades. Además, se recomienda realizar mediciones sistemáticas para ir constatando el avance y la efectividad de las actividades propuestas. Para ello se propone utilizar el procedimiento descrito por Leyva, et al., (2008).

La segunda recomendación está dirigida a desarrollar tareas de aprendizaje donde primen las estrategias instruccionales que potencien la comprensión, la retención, la aplicación y la resolución de problemas, y que permitan la transferencia (Coll et al., 1995). Todo esto encaminado a que el alumno, en el desarrollo de una misma destreza, transite por los tres niveles de desempeño cognitivo. Para lograr esto, el docente debe seguir el siguiente procedimiento:

1. Determinar la destreza a trabajar.

2. Operacionalizar la destreza en las diferentes habilidades que la componen.

3. Planificar la tarea de aprendizaje de manera que se realice una actividad, al menos, por cada habilidad correspondiente a un nivel.

Para ilustrar esta recomendación se propone un ejemplo correspondiente a la materia de Estudios Sociales del Currículo de la Educación General Básica en la República del Ecuador, en la temática de la Segunda Guerra Mundial.

1. Determinación de la destreza:

Analizar el nivel de involucramiento de América Latina en la Segunda Guerra Mundial y su participación en la fundación y las acciones de la Organización de las Naciones Unidas.

2. Operacionalización de la destreza en las habilidades:

Las destrezas son la expresión del saber hacer que se constituyen en el referente fundamental para que los docentes elaboren sus planificaciones y sus tareas de aprendizaje, MINEDUC (2011), las mismas tienen como estructura interna la habilidad, el conocimiento y el desempeño o grado de dificultad.

En este caso la habilidad es analizar, la cual significa descomponer en partes un objeto para determinar lo esencial de cada uno de ellas, considerando que en este caso el objeto es la participación de América Latina, las partes serían: causas, participantes, acciones y juicio personal. Para ello las habilidades a trabajar serían las siguientes: identificar (primer nivel), explicar (segundo nivel) y valorar (tercer nivel); una vez trabajadas estas habilidades, el estudiante podrá analizar en su conjunto el fenómeno a estudiar.

3. Planificación de la tarea de aprendizaje:

Según Verdejo y Freixas (2009), estas tareas buscan lograr el aprendizaje integrado de habilidades, conocimientos y actitudes, las cuales deben ser variadas y estar organizadas en diferentes niveles de dificultad. Un aspecto importante es el apoyo que se proporcione a los estudiantes para resolverlas, el cual va disminuyendo conforme se adapten al nivel de dificultad. De esta manera, el aprendizaje se logra de forma gradual, logrando que transiten por los tres niveles.

Continuando con el ejemplo, en la clase el docente utiliza como método el trabajo independiente del alumno bajo la dirección del docente (Klingberg, 1979), donde en la fase de orientación de la actividad, la cual desarrollará de manera escrita, indicará lo siguiente:

Leer y analizar el epígrafe correspondiente a la Segunda Guerra Mundial en el libro de texto de la materia de Estudios Sociales, Décimo Grado, páginas de la 32 a la 36.

Desarrollar las actividades de la siguiente hoja de trabajo:

1. Identifique tres de las causas que desencadenaron la Segunda Guerra Mundial.

2. Explique la causa mediante la cual América Latina se involucró en la Segunda Guerra Mundial y cuál fue su participación.

3. Valore la participación de América Latina en la Segunda Guerra Mundial.

Como se puede apreciar en este ejemplo, el docente le plantea una actividad al estudiante donde debe reproducir cuáles son las causas de la SGM (Identificar), en este caso el estudiante solo repite lo que escuchó del maestro o de otra fuente (libro) sin realizar análisis ni otro tipo de relaciones con el 
contenido; en cambio, en la segunda actividad sí debe aplicar lo aprendido para poder explicar; igual que en la tercera interrogante, donde debe aportar sus elementos y luego, a partir de ellos, crear su propio juicio valorativo, con lo que se logra que el estudiante transite por los tres niveles de desempeño.

La tercera recomendación metodológica está encaminada a la evaluación. En la actualidad existe una tendencia a realizarla mediante las llamadas pruebas objetivas, las cuales son un conjunto de preguntas claras y precisas que requieren por parte del alumno de una respuesta breve, en general, limitadas a la elección de una opción ya proporcionada (Soubiron y Camatano, 2006).

Aun cuando son llamadas objetivas no quiere decir que tengan una mayor objetividad en la evaluación, sin embargo, tienen múltiples ventajas, como son:

- Ser de fácil aplicación y corrección.

- La corrección de la prueba está menos afectada por aspectos subjetivos inherentes al corrector.

- Permite hacer diversos análisis estadísticos.

- Se pueden emplear con facilidad en las plataformas de aprendizaje on-line.

- Permiten evaluar un amplio volumen de conocimiento.

No obstante, aun cuando en la literatura se plantea que son instrumentos de medida elaborados rigurosamente, que permiten evaluar conocimientos, capacidades, destrezas, rendimiento, aptitudes y actitudes; mediante diferentes tipos de reactivos, con un alto grado de validez y fiabilidad, limitan el desarrollo cognitivo de los estudiantes, partiendo de que:

- Facilitan la memorización en detrimento del razonamiento.

- En ocasiones el azar juega un papel determinante.

- Facilitan la copia entre estudiantes.

- Establecen un determinado número de opciones que limitan el pensamiento creativo del estudiante.

Por otro lado, al darle opciones de respuesta se le está dando pistas del contenido al estudiante, él sabe que entre las opciones están las correctas, lo que le ayuda a la memorización y también a aplicar otras opciones, como son: aunque no se sepa el contenido marque opciones, de todas maneras, el estudiante sabe que no pierde nada, ¿y si acierta?, es por ello que no son recomendables y mucho menos abusar de ellas.

Estas pruebas solo permiten evaluar el primer nivel o nivel de reproducción. En este caso ya el docente le brinda cuáles son las opciones, el estudiante solo debe marcar, ¿y si el estudiante conoce otras opciones? Pero, además, en el caso de la pregunta de argumentación se puede hacer de manera objetiva, el docente le da argumentos para que ellos seleccionen, pero en este caso son los argumentos del docente ¿Dónde están los argumentos del estudiante?

De la misma manera pasa en las preguntas de valoración, ya no sería de un tercer nivel porque el estudiante no tiene que crear su juicio de valor, sino simplemente seleccionar el que le brinda el docente. Es por ello que en el diseño de las pruebas para medir los niveles de desempeño cognitivo solo se recomienda usar las preguntas de selección múltiple o elección múltiple para el primer nivel, no en el segundo y tercero.

De esta manera, combinando tareas de aprendizaje en las clases con la aplicación de pruebas donde se combinen las preguntas objetivas con las preguntas de desarrollo o ensayo se logra el tránsito por niveles en los estudiantes y su desarrollo cognitivo.

Las recomendaciones anteriores van dirigidas a los docentes, de igual manera a nivel de MINEDUC se plantea lo siguiente:

- Crear un Grupo Ministerial de Evaluación de la calidad de la Educación, el cual tendrá como objetivo analizar los resultados obtenidos para proyectar nuevas acciones que pueden estar encaminadas a:

- Elaboración de instrumentos de medición acordes a los estándares de calidad del LLECE y en función de los niveles de desempeño cognitivo.

- Aplicar periódicamente micros operativos de calidad en los cuales se aplicarán los instrumentos de medición elaborados a muestras seleccionadas aleatoriamente siguiendo los parámetros del LLECE.

- Realizar capacitaciones a los docentes sobre el desarrollo de tareas de aprendizaje y evaluaciones en función de los niveles de desempeño cognitivo.

- Regular la aplicación de pruebas objetivas.

- Incorporar la planificación de clases en función de los niveles de desempeño cognitivo. 
Un adecuado trabajo en función de los niveles de desempeño cognitivo, dirigido desde el MINEDUC, garantizará revertir los resultados obtenidos en los Estudios de la Calidad de la Educación, además de lograr aprendizajes significativos y duraderos en las nuevas generaciones de ecuatorianos, sobre la base de una Educación de Calidad.

\subsection{Discusión}

Los resultados obtenidos corroboran que existen insuficiencias en el desempeño cognitivo de los estudiantes y que es bajo el conocimiento que tienen los docentes acerca de los niveles de desempeño cognitivo y su trabajo en el aula, por lo que se hace necesario ofrecer recomendaciones metodológicas que contribuyan a resolver esta situación. En cuanto al primer objetivo, se buscó identificar el nivel de desempeño cognitivo de los estudiantes de las instituciones educativas de Santo Domingo de los Tsáchilas. Los resultados obtenidos indican un pobre desempeño cognitivo, dado que el mayor porcentaje de estudiantes $(63,21 \%)$ se ubicó en un primer nivel, siendo este el inferior. Además, solo se obtuvo un $29,26 \%$ de respuestas correctas y el $54,60 \%$ de estas corresponden al primer nivel. Resulta preocupante el porcentaje de respuestas en blanco $(21,7 \%)$.

Estos resultados coinciden con los obtenidos por Flotts, et al., (2016), el TERCE y los resultados de las pruebas PISA (2017), en donde el mayor número de estudiantes se ubicó en el primer nivel, lo que indica que se les dificulta resolver tareas en las que tienen que aplicar conocimientos científicos, analizar y resolver actividades de evaluación y creación, entre otras de nivel cognitivo superior.

Entre los factores que explican estos bajos resultados se puede inferir la preparación de los docentes para el trabajo por niveles de desempeño cognitivo (Treviño, et al., 2016). Estudios realizados por Manzano (2014, como se citó en Santiago, et al., 2017) indican que la mayoría de los docentes utilizan el $58 \%$ del tiempo del aula para exponer y explicar nuevo contenido, lo que genera un nivel de asimilación del contenido meramente reproductivo, solo el $36 \%$ del tiempo se utiliza en practicar lo aprendido y únicamente el $6 \%$ se utiliza en trabajo cognitivo de orden superior.

Similar situación se encontró en la muestra de docentes a la que se le aplicó el instrumento de medición; el $40 \%$ no conoce acerca de los estudios de calidad y el Ministerio de Educación en el Ecuador no realiza capacitaciones dirigidas al trabajo por niveles de desempeño cognitivo. Esto conlleva a que solo el $30 \%$ trabaje por niveles de desempeño cognitivo en el aula, aunque en su gran mayoría lo realizan de manera aislada.

Estos resultados coinciden con Sierra, Castellanos y García (2013), quienes caracterizaron el nivel de preparación de los docentes de la carrera de Medicina para dirigir el proceso de enseñanza-aprendizaje por los niveles de asimilación que conducen al desarrollo cognitivo de los estudiantes, quienes también constataron la insuficiente preparación pedagógica de los docentes en cuanto al conocimiento y trabajo por niveles de desempeño cognitivo.

Con el sustento en estos resultados, se procedió a elaborar las recomendaciones metodológicas para el trabajo por niveles de desempeño cognitivo en el aula, las cuales se sustentan en el desarrollo de habilidades intelectuales y la aplicación de los conocimientos en situaciones complejas, Ausubel, (1975) y el desarrollo de actividades de tipo instruccional, en las que se transite de manera integrada por los tres niveles de desempeño cognitivo. La evaluación en estas recomendaciones trasciende de ser un cuestionario de preguntas objetivas a un instrumento desarrollado en función de las habilidades que se han ido formando.

Además, la evaluación, lejos de ser un acto reduccionista, pasa a ser un proceso en el que se combinan las pruebas con otro tipo de técnicas de evaluación como las exposiciones orales, los estudios de caso y los informes de investigación (Rossi, et al., 2009). Es importante destacar las limitaciones del estudio, las cuales se centran en el tamaño de la muestra, para ello se recomienda que sea ampliada en estudios futuros, tanto en docentes como en estudiantes.

\section{Conclusiones}

Los resultados obtenidos a partir de la aplicación de tres instrumentos a una muestra de 117 estudiantes de instituciones educativas del Cantón Santo Domingo evidenciaron un insuficiente nivel de desempeño cognitivo, resultando preocupante el bajo número de respuestas correctas obtenidas y que estas se sitúan en el primer nivel de desempeño, lo que indica que los estudiantes tienen dificultades para aplicar los conocimientos adquiridos en la resolución de problemáticas que se le presentan. 
En cuando al nivel de conocimiento que tienen los docentes de las instituciones educativas del Cantón Santo Domingo acerca del trabajo por niveles de desempeño cognitivo, se evidenció que es bajo, se dedica la mayor parte del tiempo de trabajo en el aula a desarrollar actividades de orden cognitivo inferior o reproductivo y el Ministerio de Educación en el Ecuador no difunde los resultados obtenidos a nivel de país en los estudios de calidad a nivel regional.

Los resultados obtenidos en los estudios de calidad de la educación y el insuficiente conocimiento que tienen los docentes acerca del trabajo por niveles de desempeño cognitivo en el aula requieren de un conjunto de recomendaciones que van encaminadas al diagnóstico permanente del nivel alcanzado por los estudiantes, que las actividades y experiencias de aprendizaje que se ejecuten en el aula les permitan a los estudiantes transitar por los diferentes niveles, diseñar instrumentos pertinentes con el nivel de desempeño cognitivo que se quiere evaluar y disminuir el uso de las pruebas objetivas y la creación de una entidad ministerial encargada del monitoreo y seguimiento de la calidad de la educación en el país.

Se ofreció un conjunto de recomendaciones metodológicas para el trabajo por niveles de desempeño cognitivo sustentadas en el paradigma cognitivo o de procesamiento de la información, encaminadas a potenciar el desarrollo de habilidades intelectuales que permitan aplicar los conocimientos a situaciones complejas. Estas recomendaciones se basan en el diseño de actividades de tipo instruccional y una evaluación procesual, donde la evaluación del desempeño cognitivo no se centre en la aplicación de pruebas objetivas, sino que diseñen y apliquen otras técnicas e instrumentos y actividades de evaluación (proyectos, disertaciones orales, estudios de caso e informes de investigación, entre otros) que garanticen la verificación del tránsito de los estudiantes por los diferentes niveles de desempeño cognitivo.

\section{Referencias}

Albornoz Zamora, E. J., \& Guzmán, M. C. (2016). Desarrollo cognitivo mediante estimulación en niños de 3 años. Centro desarrollo infantil Nuevos Horizontes. Quito, Ecuador. Universidad y Sociedad, volumen (8), pp. 186-192. Recuperado el 5 de marzo de 2020, de http:// scielo.sld.cu/scielo.php?pid=S2218-36202016000400025\&script=sci_arttext\&tlng=pt.

Alves, A. F.; Assis, C. M.; Martins, A. \& da Silva, L. (2017). Cognitive performance and academic achievement. How do family and School converge. European Journal of Education and Psychology, (10), pp. 49-56. doi: https://doi.org/10.1016/i.ejeps.2017.07.001

Ausubel, D. P. (1975). Psicología educativa. Un punto de vista cognoscitivo. México. Trillas.

Barragán Castrillón, Bernardo (2007). Del análisis de la transmisión al análisis de la construcción: la emergencia del paradigma cognitivo en la educación en Colombia. Revista Electrónica Actualidades Investigativas en Educación, 7(3). doi: https://doi.org/10.15517/aie.v7i3.9281

Barrezueta, A. E. T., Chiriboga, E. A. V., Mera, G. M. M., \& Andina, M. J. Z. (2019). Desarrollo cognitivo y funcionalidad familiar de infantes en las comunidades urbano-marginales de Ecuador. Revista de ciencias sociales, 25(1). Recuperado el 1 de mayo de 2020, de https://dialnet.unirioja.es/servlet $/$ articulo?codigo $=7113733$

BBC Mundo, Agencias Redacción (2019, 12/17/2019). Las claves de los mejores sistemas educativos del mundo. Recuperado 17 de diciembre de 2019, de https://www.eluniverso.com/noticias/2019/12/17/nota/7648094/claves-mejores-sistemas-educativos-mundo

Coll, Cesar. (1995). El constructivismo en el aula. Barcelona: Graó.

Cooper, D. (1990). Cómo mejorar la comprensión lectora. Madrid: Visor.

Davis, L. L. (1992). Instrument review: Getting the most from a panel of experts. Applied Nursing Research, (5), pp. 194-197. doi: https://doi.org/10.1016/S0897-1897(05)80008-4

De Vega, M. (1984). Introducción a la psicología cognitiva, Madrid: Alianza.

Feldberg, C., Stefani, D., Tartaglini, M. F., Hermida, P. D., García, L. M., Somale, M. V., \& Allegri, R. (2020). La influencia de la educación y la complejidad laboral en el desempeño cognitivo de adultos mayores con deterioro cognitivo leve. Ciencias Psicológicas, 14(1), e2194. doi: https://dx.doi.org/22235/cp.v14i1.2194

Flotts, P., Manzi, J., Jiménez, D., Abarzúa, A., Cayuman, C., \& García M. J. (2016). Tercer Estudio regional y Comparativo de la Calidad de la Educación. Logros de aprendizaje. (Informe de resultados). TERCE. Recuperado el 1 de febrero de 2020, de http://unesdoc.unesco.org/images/0024/002435/243532S.pdf 
Gardner, H. (1987). La nueva ciencia de la mente: Historia de la psicología cognitiva. Barcelona: Paidós.

Gutiérrez, O. (2014). Tendencias en la evaluación de los aprendizajes, Revista de Teoría y Didáctica de las Ciencias Sociales, (9), pp. 111-130.

Ineval (2014). Terce: Ecuador mejora sus resultados. Publicaciones Ineval. Recuperado el 12 de diciembre de 2019, de http://www.evaluacion.gob.ec/wp-content/uploads/downloads/2016/06/DAGI_Terce14_InformeEjecutivo_20141203.pdf

Klingberg, L. (1979). Introducción a la Didáctica General. La Habana: Pueblo y Educación.

Laboratorio Latinoamericano de Evaluación de la Calidad de la Educación (2015). Informe de resultados del Tercer Estudio Regional y Comparativo de la Calidad de la Educación. Logros de Aprendizaje. Recuperado el 2 de enero de 2020, de http://unesdoc.unesco.org/images/0024/002435/243532S.pdf

Lawshe, C. H. (1975). A quantitative approach to content validity. Personnel Psychology, 28, pp. 563-575. doi: https://doi.org/10.1111/j.1744-6570.1975.tb01393.x

Leyva, L. M., Proenza, Y., Leyva, J. L., Cristo, R. \& Romero, R. (2008). Reflexiones sobre la evaluación de la calidad del aprendizaje en la práctica pedagógica en la escuela primaria. Revista Iberoamericana de Educación, (44). Recuperado el 10 de marzo de 2020, de https://rieoei.org/historico/deloslectores/1922Leyva.pdf

MINEDUC (2011). Actualización y fortalecimiento curricular en la Educación General Básica 2010. Quito.

Nickerson, R. S., Perkins, D. N. \& Smith. E. E. (1987). Enseñar a pensar. Aspectos de la aptitud intelectual. Barcelona: Paidós.

Papalia, D. E., Duskin Feldman, R., Martorell, G., Berber Morán, E., Vázquez Herrera, M., Ortiz Salinas, M. E., \& Javier Dávila, J. F. (2012). Desarrollo bumano: D. E. Papalia, R. Duskin Feldman \& G. Martorell; revisado por E. Berber Morán \& M. Vázquez Herrera; traducido por M. E. Ortiz Salinas \& J. F. Javier Dávila (12a ed.). México D. F.: McGraw Hill.

Pozo, J. I. (1989). Teorías cognitivas del aprendizaje. Madrid: Morata.

Puig, S. (2003). Una aproximación a los niveles de desempeño cognitivo. La Habana: ICCP.

Puig, S. (2009). Propuesta para la evaluar el desempeño cognitivo de los escolares. La Habana, Editorial Academia.

Santiago, R., Díez, A., \& Andía, L. A. (2017). Flipped classroom. 33 experiencias que ponen patas arriba el aprendiraje. Editorial UOC (Oberta UOC Publishing, SL) Barcelona.

Rossi, A. P., Di Prátula, H. R., \& Vitale, A. J. (2009). Evaluación del proceso de enseñanza-aprendizaje sobre fuentes renovables de energía desde los paradigmas cognitivo y ecológico-contextual. Formación universitaria, 2(4), pp. 15-22. doi: https://doi.org/10.4067/S0718-50062009000400003

Rubio, R. et al. (2006). Los niveles de asimilación y niveles de desempeño cognitivo. Reflexiones. Revista Humanidades Médicas, Vol. 6, n. ${ }^{\circ}$ 16, enero-abril. Recuperado el 22 de noviembre de 2019, de http://scielo.sld.cu/scielo.php?script=sci_arttext\&pid=S1727-81202006000100005

Ruvalcaba, H. (2015). Los niveles de asimilación del contenido y la práctica docente. Iquique: UNAP. Recuperado el 12 de diciembre de 2019, de http://www.unap.cl/prontus unap/site/artic/20111013/asocfile/20111013115255/los_niveles_de_asimilaci_n_del_contenido_y_la_practica_docente.pdf

Sierra, Y., Castellanos, E., \& García, Y. (2013). Preparación de los docentes para dirigir el proceso de enseñanza aprendizaje utilizando niveles de asimilación. EduMeCentro, 5(3), pp. 95-107.

Soubiron, E. \& Camatano, S. (2006). Diseño de pruebas objetivas. Universidad de la República, Uruguay. Recuperado el 11 de enero de 2020, de https://web.ua.es/es/ice/documentos/recursos/materiales/ev-pruegas-objetivas.pdf

Terranova, A., Viteri, E., Medina., Mera., Treviño, E., Fraser, P., Meyer, A., Morawietz, A., Inostroza, P. \& Naranjo, E. (2016). Factores asociados. Tercer Estudio Regional Comparativo y Explicativo. Laboratorio Latinoamericano de Evaluación de la Calidad de la Educación OREAL/UNESCO, Chile. Recuperado el 12 de noviembre de 2019, de https://unesdoc.unesco.org/ark:/48223/pf0000243533

Tundidor-Bermúdez, Á. M. (2019). Hacia una reclasificación de los niveles de asimilación del conocimiento. FEM: Revista de la Fundación Educación Médica, 22(4), pp. 197-197. doi: https://doi.org/10.33588/fem.224.1006

Verdejo, P., Freixas, R. (2009). Educación para el pensamiento complejo y competencias: Diseño de tareas y experiencias de aprendiraje. México: Innovacesal.

Villalobos, J. (2003). El docente y actividades de enseñanza/aprendizaje: algunas consideraciones teóricas y sugerencias prácticas. Educere, 7(22), pp. 170-176. 